\title{
Targeted Regulation of Cariogenic Pathogen Streptococcus mutans in Biofilms by Pheromone-Guided Antimicrobial Peptides
}

Li $\mathbf{Y H}^{1,2^{*}}$, Li $\mathbf{M}^{1,3}$ and Tian $\mathbf{X L}^{1}$

${ }^{1}$ Department of Applied Oral Sciences, Dalhousie University, Halifax, NS, Canada

${ }^{2}$ Department of Microbiology and Immunology, Dalhousie University, Halifax, NS, Canada

${ }^{3}$ Lanzhou University, Lanzhou, Gansu, China

*Corresponding author: Li YH, Department of Applied Oral Sciences, Dalhousie University, Halifax, Nova Scotia, Canada, Tel: 1-902-494-3063; E-mail: yunghua.li@dal.ca

Received date: June 06, 2018; Accepted date: July 24, 2018; Published date: July 31, 2018

Copyright: $\odot 2018 \mathrm{Li} \mathrm{YH}$, et al. This is an open-access article distributed under the terms of the Creative Commons Attribution License, which permits unrestricted use, distribution, and reproduction in any medium, provided the original author and source are credited.

\begin{abstract}
A major challenge using wide-spectrum antibiotics to treat an infectious disease in a natural ecosystem, such as in the oral cavity that contains the resident microflora, is that such therapy may result in ecological disruption and negative clinical consequences. Therefore, development of target-specific antimicrobials that selectively target specific pathogens may provide an opportunity to circumvent the problem. By exploiting the fact that a quorum sensing signal molecule can specifically bind to the same bacterial species at a nano-molar concentration, researchers in several laboratories have developed a new class of antimicrobials, called pheromone-guided antimicrobial peptides. The rationale of creating such antimicrobial peptides is based on the addition of a targeting domain of a bacterial quorum-sensing signal molecule to the killing domain of a known antimicrobial peptide. Both functional domains are fused to generate a new fusion peptide without detrimental change of their activities. The major advantage of such peptides is that the targeting domain can guide these fusion molecules to bind selectively to the target pathogen, leading to selective killing or quorum-sensing interference.
\end{abstract}

Keywords: Microbial biofilms; Streptococcus mutans, Antibiotic resistance; Quorum sensing; Quorum sensing inhibitor; Pheromoneguided antimicrobial peptides

\section{Introduction}

The human oral cavity is a complex ecosystem that harbors hundreds species of microbes, which are largely living on the tooth surfaces as dental biofilms [1-3]. Species diversity, high cell density and close proximity of microbial cells are typical of life in dental biofilms, where microorganisms interact with one another and develop complex interactions $[2,3]$. In healthy individuals with the right dietary and oral hygiene habit, the oral microbiota lives in symbiosis with the host [1]. The resident species of microbes in dental biofilms can maintain a relatively stable and harmless relationship with the host, despite frequent exposure to environmental perturbations and host physiological factors $[1,4]$. It is well known that such stability or the homeostasis results from a dynamic balance of microbial-microbial and microbial-host interactions [4]. When the ecological homeostasis is not significantly disrupted, the resident microbes in dental biofilms usually promote the oral health by stimulating the immune system and by preventing invasion of pathogenic species [3]. Under some circumstances, however, the homeostasis may break down, leading to microbial shifts to an imbalanced status or dysbiosis, which predisposes a site to dental diseases [4].

Streptococcus mutans is a Gram-positive bacterium that depends on a "biofilm lifestyle" for survival and persistence in dental biofilms $[1,3]$. Under certain conditions, $S$. mutans can rapidly produce acids from fermentable dietary carbohydrates and lower the biofilm $\mathrm{pH}$, which initiates demineralization of the tooth surface or dental caries [4]. $S$. mutans is therefore considered as a primary etiological agent of dental caries worldwide [5]. S. mutans can also be a cause of dental-related bacteremia and subacute infective endocarditis [5]. Although dental caries is not a life-threatening disease, diagnosing, treating and retreating caries and caries-related diseases is still a major financial burden to public health systems worldwide [6]. Clinically, eliminating $S$. mutans from dental biofilms using wide-spectrum antimicrobial agents is not practical, because these agents inevitably result in indiscriminate killing of the resident microbiota, leading to ecological disruption and other clinical negative consequences [7-9]. Therefore, prevention of $S$. mutans and its associated diseases requires the development of new strategies that specifically target $S$. mutans without significant disruption of other species in dental biofilms.

In recent years, several attempts have been made to achieve targetspecific antimicrobial therapy by coupling antibiotics to speciesspecific monoclonal antibodies or by creating fusion peptides that combine killing domains with bacterial recognition domains [9-14]. These narrow-spectrum antimicrobials can selectively target specific pathogens with relatively little effect on the other members of the resident microflora. Among these antimicrobials with "targeting activity", pheromone-guided antimicrobial peptides or PG-AMPs have shown the greater effectiveness and selectivity for targeting specific pathogens. Such antimicrobial molecules consist of a targeting domain of a quorum-sensing signal molecule from a bacterial pathogen and the killing domain of a known antimicrobial peptide $[11,15]$. Both functional domains are fused via a short linker to generate a new fusion peptide without detrimental change of their functional activities.

The major advantage of such PG-AMPs is that the targeting domain can guide the fusion molecule to bind selectively to the target pathogen, leading to selective killing. In particular, such PG-AMPs can 
achieve a high efficacy for targeted killing at a very low concentration, since the bacterial recognition domain, such as quorum-sensing signal pheromone, can selectively bind to the same species at a nano-molar concentration [16]. These PG-AMPs have been successfully developed against various bacterial pathogens, including MRSA Staphylococcus aureus, vancomycin-resistant Enterococcus faecalis, Pseudomonas spp, and cariogenic pathogen $S$. mutans [11-16]. Therefore, PG-AMPs have added an exciting opportunity for development of new antimicrobials that selectively target specific pathogens. However, PG-AMPs and their application as an alternative antimicrobial therapy are still in their infancy.

This review briefly summarizes the current advance in this field and raises questions for further study.

\section{Rational Design and Structure-Activity Analysis}

The design and synthesis of PG-AMPs is based on the rationale that the killing domain of a known antimicrobial peptide is fused via a small linker to the high-affinity targeting domain of a quorum sensing signal pheromone from a bacterium (Figure 1).

Therefore, all PG-AMPs are chimeric molecules that largely depend on chemical synthesis followed by experimental confirmation of their selective killing activity against target pathogens. Thus, design and synthesis of PG-AMPs requires previous identification of two functionally independent molecules, the killing domain of a known AMP and the targeting domain of a bacterial quorum sensing signal pheromone [13-16].

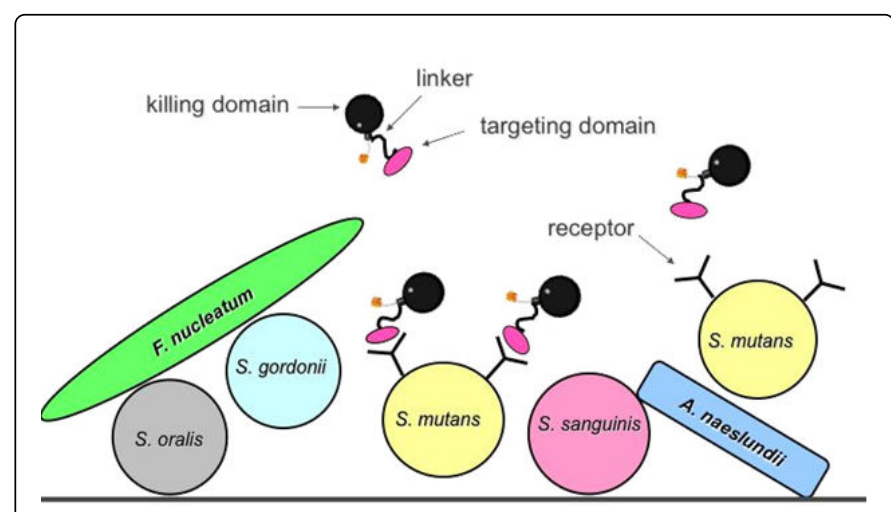

Figure 1: A schematic diagram describes the rationale of a PG-AMP to achieve selective killing of a target bacterium, such as $S$. mutans, in a multi-species microbial community. PG-AMPs, such as IMB-2 and HP30, are fusion peptides consisting of a targeting domain of a quorum-sensing signal peptide or CSP from $S$. mutans and the killing domain of a marine-derived antimicrobial peptide. The two functional domains are fused via a short linker to generate a fusion peptide. In such a way, the targeting domain can guide the fusion peptides to bind selectively to $S$. mutans, thereby, triggering selective killing or quorum sensing interference.

However, previous knowledge of two functional domains may not be sufficient to predict targeting and killing activities of a newly constructed fusion peptide [16]. In many cases, such fusion molecules may be lack of an expected functionality because of changes in primary sequences, charge and hydrophobic features of a new fusion molecule. In few cases, a linker sequence may also affect targeting activity of a fusion molecule [13]. Therefore, it is important to design and synthesize an array of PG-AMPs for antimicrobial screening based on their structure-activity relationships obtained by NMR spectroscopy analysis [16-18].

Generally, a library that contains a list of proposed fusion molecules needs to be constructed and experimentally evaluated for their killing activity and selectivity. Based on this rationale, Mai et al. have constructed a library of fusion peptides consisting of the killing domain of a marine-derived AMP [19] and the targeting domain of a competence signaling peptide or CSP from $S$. mutans, a primary cariogenic pathogen $[16,20]$. By screening the fusion peptide library, these researchers have identified several pheromone-guided AMPs that selectively target and kill $S$. mutans [16]. The active fusion peptides consist of three parts: an 8-residue targeting domain of signal peptide CSP, a GG linker and the killing domain of a marine-derived AMP (Table 1).

Structural analysis by NMR spectroscopy confirms that the active PG-AMPs, such as IMB-2 and HP30, form an amphipathic, a-helical character, which appears to favor the interaction of these peptides with the ComD receptor in the cell membrane of $S$. mutans, whereas inactive peptides adopt a relatively unstructured, random coil form (Figure 2). In another study, $\mathrm{He}$ et al. have developed a strategy to design two libraries of fusion peptides that also target $S$. mutans by a tunable, building-block approach that use various combinations of an antimicrobial, a targeting and a linker $[17,18]$. This approach enables the researchers to modify and improve the targeted killing activities of fusion peptides in the peptide libraries.

In both studies, the newly constructed peptides are optimized via modification of their primary sequences, such as $\mathrm{C}$-terminal amidation to reduce the end charge or residue substitution to enhance the effectiveness and stability in various physiological conditions, such as salt concentrations, $\mathrm{pH}$, and proteolytic activity in blood or other body fluids [16-18,21].

\section{Evaluation of Targeted Killing Activity}

All PG-AMPs newly constructed have to be experimentally tested and validated for their selective killing activities against target pathogens. The in vitro effectiveness and selectivity of a targeted peptide are usually evaluated by experimental tests under several growth conditions. For example, both IMB-2 and HP30 show strong bactericidal activity (MIC 2.8 and $2.5 \mu \mathrm{M}$ ) against $S$. mutans under a mono- or dual-species planktonic culture $[16,20]$. However, this may not be sufficient to determine the selectivity of these fusion peptides, so that these fusion peptides are further tested in multi-species cultures or in biofilms, which contain closely related oral species, such as $S$. sanguinis, $S$. gordonii or other species found commonly in dental biofilms [16,21]. These studies confirm that both IMB-2 and HP30 selectively target $S$. mutans in the presence of these closely related species under tested culture conditions. The data suggest that the binding of these PG-AMPs to $S$. mutans cells is species-specific, since a strong killing activity against $S$. mutans is clearly observed in monoand dual-species cultures [21]. In particular, these peptides show a dose-dependent selective killing of $S$. mutans in biofilms, although the higher concentrations are required to achieve desirable killing. In another study, a targeted antimicrobial peptide, G10KHc, exhibits a high degree of the specificity for killing bacterium Pseudomonas mendocina [13]. This peptide can bind tightly to the surface of the target species, resulting in rapid and selective killing of the pathogen in a mixed culture condition. It has been found that the targeting domain 
Citation: Li YH, Li M, Tian XL (2018) Targeted Regulation of Cariogenic Pathogen Streptococcus mutans in Biofilms by Pheromone-Guided Antimicrobial Peptides. J Antimicrob Agents 4: 175. doi:10.4172/2472-1212.1000175

Page 3 of 6

(KH) and killing domain (G10) of G10KHc function separately in such a way that the targeting domain of G10KHc selectively delivers the peptide to the $P$. mendocina surface, whereas the killing domain then rapidly disrupts the outer membrane and increases its ability to access the cytoplasmic membrane to cause cell death. The evidence from this study suggests that the specificity of $\mathrm{G} 10 \mathrm{KHc}$ against $P$. mendocina results from an independent activity of the $\mathrm{KH}$ targeting domain [13]. The combination of these independent processes results in large overall enhancements in the killing activity and selectivity against the target pathogen.

\begin{tabular}{|l|l|l|l|l|}
\hline Name of Peptide & Fusion Peptide (Targeting domain-Linker-Killing domain) & MIC & Target Organism & Ref. \\
\hline PMC-SA & (agrD1)-colicin la (175 residues) & $4 \mu \mathrm{g} / \mathrm{ml}$ & MSSA or MRSA Staphylococcus aureus & 12 \\
\hline G10KHc & KNLRRIIRKGIHIIKKYG(G10)-GGSGGS-KKHRKHRKHRKH(KH) & $2.8 \mu \mathrm{M}$ & Pseudomonas mendocina & 13 \\
\hline CSP-18* & SGSLSTFFRLFNRSFTQA & NA & Streptococcus mutans & 23 \\
\hline CSP-21* & SGSLSTFFRLFNRSFTQALGK & NA & S. mutans & 20 \\
\hline C16G2 & TFFRLFNRSFTQALGK -GGG- KNLRHRKGIHIIKKY & $5.2 \mu \mathrm{M}$ & S. mutans & 15 \\
\hline M8-G2 & TFFRLFNR -GGG- KNLRIRKGIHIIKKY & $3.25 \mu \mathrm{M}$ & S. mutans & 15 \\
\hline IMB-1 & TFFRLFNR-GG- GWGSFFKKAAHVGKHVGKAALTHYL NH2 & $2.2 \mu \mathrm{M}$ & S. mutans & 16 \\
\hline IMB-2 & TFFRLFNR-GG- GWGSFFKKAAHVGKL NH2 & $2.8 \mu \mathrm{M}$ & S. mutans & 16 \\
\hline IMB2-H/S14 & TFFRLFNR-GG- GWGHFFKKAAHVGKL NH2 & $2.8 \mu \mathrm{M}$ & S. mutans & 16 \\
\hline HP30 & SGSLSTFFRLFNR-GG- GWGHFFKKAAHVGKL NH2 & $2.5 \mu \mathrm{M}$ & S. mutans & 21 \\
\hline CSP-18: Comp &
\end{tabular}

CSP-18*: Competence-stimulating peptide from S. mutans after C-terminal cleavage by SepM; CSP-21: CSP before the C-terminal cleavage. Underlined is minimal targeting sequence from CSP. NH2: C-terminal amidation. H/S14: a His-to-Ser14 substitution (bold). MIC: minimum inhibitory concentration. NA: not applicable.

Table 1: Examples of pheromone-guided antimicrobial peptides (PG-AMP).

A

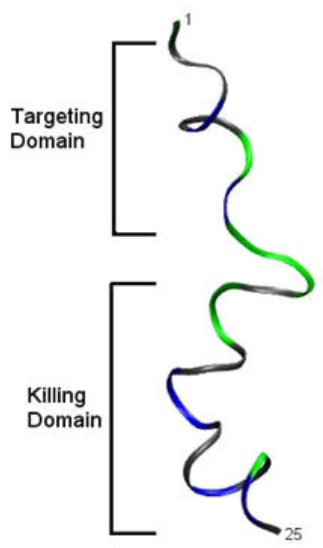

B

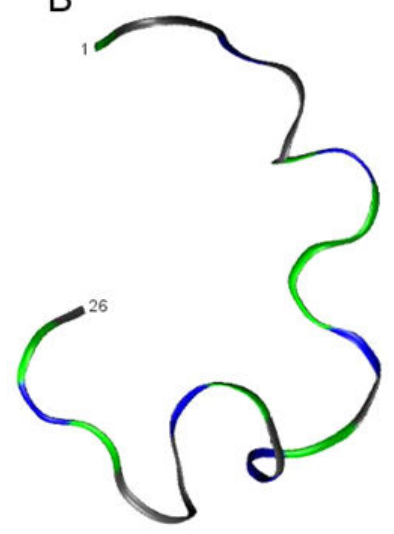

Figures $2(\mathrm{~A})$ and $2(\mathrm{~B})$ : The diagrams represent two reconstructed fusion AMPs, IMB-2 2(A) and IMB-3 2(B), which are the lowest energy structures based on NMR spectroscope analysis. IMB-2 (an active killing peptide) forms an amphipathic $\alpha$-helical structure, whereas IMB-3 (an inactive killing peptide) is random coiled and unstructured. Each of the fusion peptides consists of an 8-residue targeting domain of $S$. mutans CSP and the killing domain of a marine-derived AMP. Two functional domains are fused via a short linker to generate a new fusion peptide. Adapted from Mai et al. $[16]$.

\section{The Action Mechanisms}

Theoretically, pheromone-guided AMPs should function to exhibit two types of activities, the targeting activity that guides a fusion peptide to bind selectively to the target pathogen, and bactericidal activity that triggers rapid killing by a mechanism that primarily depends on the killing domain. However, the action mechanisms for targeted killing of PG-AMPs are not fully understood. Each new PGAMP requires experimental confirmation of its action mechanism. Currently, two types of the action mechanisms may be involved in the activities of PG-AMPs.

\section{Killing by Disrupting the Cell Membrane}

Kaplan et al. have described the action mechanism of a pheromoneguided AMP, C16G2, which contains a portion of the $S$. mutans CSP (CSP16) and is designed to target $S$. mutans [22]. By comparing C16G2 with a known AMP melittin B, these researchers confirm that C16G2 has a selective membrane-disrupting activity that results in loss of membrane potential $(\Delta \Psi)$ of $S$. mutans, leading to cell death at a level of potency similar to melittin B. However, the exact mechanism how C16G2 selects $S$. mutans remains unclear, since the ComD receptor that is supposed to be the binding site for C16G2 has no role in targeting of C16G2 to the surface of $S$. mutans. The evidence suggests that C16G2 may involve the membrane-binding or partition steps that enable C16G2 to retain on the $S$. mutans surface, independently of the ComD receptor [22]. However, Mai et al. have found that a PG-AMP, IMB-2, consisting of eight residue targeting domain of CSP appears to enable the fusion peptide to bind to the ComD receptor of $S$. mutans, because deletion of comD results in significant reduction in killing of $S$. mutans by IMB-2. In other words, the target domain of IMB-2 
appears to play a true role in guiding the peptide to bind selectively to $S$. mutans cells, triggering killing by the killing domain.

More recently, Tian et al. have tested a newly constructed peptide HP30, which was designed to have five extra residues, SGSLS, at its Nterminal arm that is identical to that of CSP [21]. They have found that HP30 shows an improved selectivity to target $S$. mutans cells for killing, which likely results from an improved binding of HP30 to the ComD receptor protein of $S$. mutans. This divergence was not clear until a recent discovery that an extracellular protease SepM on $S$. mutans cell surface is required for the processing of 21-residue peptide to generate an 18-residue CSP by cleavage of C-terminal three residues LGK [23]. Since the targeting domain of IMB-2 and HP30 does not have the C-terminal three residues LGK, this implies that the Cterminal killing domain of IMB-2 and HP30 would not be subject to the cleavage by protease SepM (Table 1). In contrast, C16G2 contains the intact C-terminal residues of CSP (CSPC16), including LGK, suggesting that the $\mathrm{C}$-terminal killing domain of $\mathrm{C} 16 \mathrm{G} 2$ can be cleaved off by protease SepM, releasing two independent peptides, the targeting and the killing domains. The discovery of the protease SepM explains the divergence in the action mechanisms between C16G2 and IMB-2 or HP30. Thus, the action mechanism of C16G2 observed by Kaplan et al. can be interpreted as the action mode of the C-terminal killing domain alone, which is actually consistent with their observation in the report [22]. However, this does not exclude the possibility that both IMB-2 and HP30 may involve an additional unknown binding site on the surface of $S$. mutans, since they also kill $15 \%-20 \%$ of the ComD deletion mutant cells. It is speculated that both IMB-2 and HP30 may interact mainly with the ComD receptor and, to a less extent, with the cell membrane of $S$. mutans before triggering the killing.

\section{Inhibition by Quorum Sensing Interference (QSI)}

PG-AMPs are chimeric molecules consisting of the binding domain of a quorum sensing signal molecule and the killing domain of a known antimicrobial peptide [13-16]. The binding domain recognizes and interacts with the cognate receptor on a target pathogen. However, it remains an open question whether the binding of such a fusion peptide to its cognate receptor affects quorum-sensing signaling and its regulated group activities. Recent work by screening a library of PGAMPs reveals that some fusion PG-AMPs initiate rapid killing and these peptides are often selected for further investigation [16,21]. However, the work also shows that some fusion peptides display "slow killing" or inhibition of the target organism [21]. Since the short-time killing is not so great, these fusion peptides are often not included for further study.

We have recently investigated some of these "slow killing" peptides and found that some peptides show quorum sensing inhibition, as detected by luciferase reporter assay of the promoters of quorum sensing inducible genes (unpublished data). The initial work suggests that these fusion peptides may exhibit dual activities, quorum sensing inhibition and targeted killing, although the mechanisms behind these observations remain to be determined. It is then reasonable to assume that fusion peptides that do not confer rapid killing may affect the target organism by other mechanisms, which requires further investigation in future study. In recent years, quorum-sensing mechanisms have been recognized as a new target for development of quorum sensing inhibitors as alternative antimicrobials [22-25]. Any compound that interferes with interactions between quorum sensing signal molecules and their cognate receptors might block bacterial cell- cell communication and its controlled virulence. QSI that specifically blocks or overrides bacterial quorum sensing can make the pathogens lose their ability to communicate for coordinated activities. It is believed that QSI-interfered pathogens can be readily eliminated by the host defense mechanisms [24]. This approach potentially has several advantages, including exerting less selective pressure toward resistant microbes, preserving the normal microflora, and expanding the repertoire of bacterial targets [25]. Clearly, further study is required to elucidate the action mechanisms of individual PG-AMPs.

\section{The Stability in Physiological Conditions}

It is known that many AMPs are sensitive to variations in salt concentrations, ionic strength, $\mathrm{pH}$ and proteolytic activity in body fluids, such as serum, saliva and gastro-intestinal fluid, resulting in reduction or loss of their bacteriocidal activities [26]. Therefore, the ability to resist physiological salts, $\mathrm{pH}$ fluctuation and proteolytic activity is crucial for pheromone-guided AMPs to retain their killing activities in the host. Both IMB-2 and HP30 that specifically target $S$. mutans have been assessed for their stability in physiological body fluids, such as human saliva $[16,21]$.

Human saliva is a mixed fluid that contains $99 \%$ water and $1 \%$ organic and inorganic molecules with an average $\mathrm{pH} 6.7$ [27]. Total electrolytes in saliva largely account for ionic strength or salt concentrations, which are normally lower than those in serum but vary greatly with saliva flow and the host health status. In addition, whole saliva contains a number of components, such as antimicrobial compounds, proteins, glycoproteins and bacterial products, which account for most of its proteolytic activity [27]. It is reported that both IMB-2 and HP30 are capable of maintaining killing activity in human saliva for 30 minutes, which is sufficient for the peptides to exhibit nearly full killing activity, indicating the relative stability of these fusion peptides in human saliva [16,21]. It is also found that a low dose of chelating agent EDTA can significantly improve the stability of these peptides in human saliva, suggesting that it is an advantage by using a PG-AMP combined with a low concentration of EDTA. In addition to resistance to physiological salt and proteolytic activity, IMB-2 and HP30 are also found to be relatively resistant to low $\mathrm{pH}[16,28]$. In particular, a modification of the peptide by a Ser-to-His substitution, named IMB2-H/S14, improves the killing activity of the peptide by $10-20 \%$ at lower $\mathrm{pH}$ levels [16]. Thus, IMB-2H/S14 appears to be more desirable than IMB-2 for application under $\mathrm{pH}$ fluctuation conditions such as in dental plaque biofilms.

\section{Targeted Regulation of $\boldsymbol{S}$. mutans in Saliva-Derived Microbial Community}

Dental biofilm is a microbial community that is usually characterized by a remarkable stability among the component species, despite regular exposure to perturbations of host physiological factors [1-3]. Interestingly, such stability or the homeostasis in dental biofilms is often associated with a healthy oral condition $[1,3]$. A sudden change in relative abundance of one or a few species in dental biofilms can result in the breakdown of homeostasis, called dysbiosis, in the community, predisposing the site to a disease [3]. For example, frequent consumption of fermentable dietary carbohydrates in the oral cavity may favor the overgrowth of sugar-fermenting and acidproducing-and-resistant bacteria (Figure $3 \mathrm{~A}$ ), such as $S$. mutans and Lactobacillus spp in dental biofilms [1,3-5]. The dominance by acidproducing-and-resistant $S$. mutans in dental biofilms leads to the 
breakdown of the homeostasis, predisposing the site to tooth decay [3-5]. Recent studies have explored the possibility of using PG-AMPs as a target-specific strategy to remove $S$. mutans from dental biofilms and to restore the balance of the community by providing a competitive advantage to non-cariogenic bacteria in the community (Figure 3B). During evaluation of the selectivity of PG-AMPs, a major challenge is how a PG-AMP, such as IMB-2, HP30 or C16G2, could achieve the effective concentration and maintains its selectivity for killing in multi-species microbial community or biofilms [29]. This question clearly requires in vivo tests or in an in vitro model system that closely mimics physiologically relevant oral microbiota.

Despite this challenge, a study has been conducted to evaluate the targeted killing and selectivity of a PG-AMP in such an in vitro model system [30]. In particular, a saliva-derived microbial community that consists of physiologically relevant oral bacteria has been developed and proved to be a useful tool for screening new antimicrobials [30]. As technology advances in engineering of microbial community along with microbiome analysis, it becomes possible to construct such an in vitro microbial community for precise evaluation of the efficacy of PGAMPs.

The advantages of using this model system are that such an in vitro microbial community is relatively easy to manipulate in a laboratory. Microbial species in the community can be quantitatively analyzed and the data are highly reproducible [30]. For this reason, Guo et al. investigated the efficacy of C16G2 against $S$. mutans in a saliva-derived microbial community spiked with $S$. mutans. The target community is allowed to recover after 24 hours at $37^{\circ} \mathrm{C}$ for evaluation of this peptide against $S$. mutans in a multi-species community.

Treatment with C16G2 for 30 minutes resulted in approximately $10^{6}$-fold greater reduction in viable cell count of $S$. mutans compared with the carrier control in the community. In contrast, the total bacterial count was relatively unaffected and only decreased by $<10-$ fold [29]. Their work suggests that C16G2 is capable of targeted removal of $S$. mutans from multi-species cultures with minimal effect on non-cariogenic streptococci. The findings suggest that targeted removal of $S$. mutans could restore the balance of the community by providing an advantage to non-cariogenic bacteria. This work suggests a potential application of PG-AMPs in modulating community ecology.

\section{Clinical Application and Efficacy}

Currently, very few of study have been carried out to investigate the efficacy and clinical application of PG-AMPs in dealing with an infectious disease. There is considerable challenge in clinical application, because of some doubts about the ability of PG-AMPs, like many other antimicrobial peptides, to achieve effective bactericidal activity under physiological conditions; the susceptibility of peptides to proteolytic degradation; the lack of information about potential toxicities in vivo; and comparatively high costs associated with peptide production. Despite such challenges, a pilot study has been conducted in human volunteers to evaluate clinical efficacy of a PG-AMP, C16G2, against $S$. mutans by a mouth rinse [31].

It has been found that C16G2 is considerably effective in stopping the growth of $S$. mutans in dental biofilms and reduces the formation of total biofilms during the course of the study. As a proof-of-concept, this study has achieved a relatively good result in terms of the selectivity, delivery method and stability of the peptide in the human host.

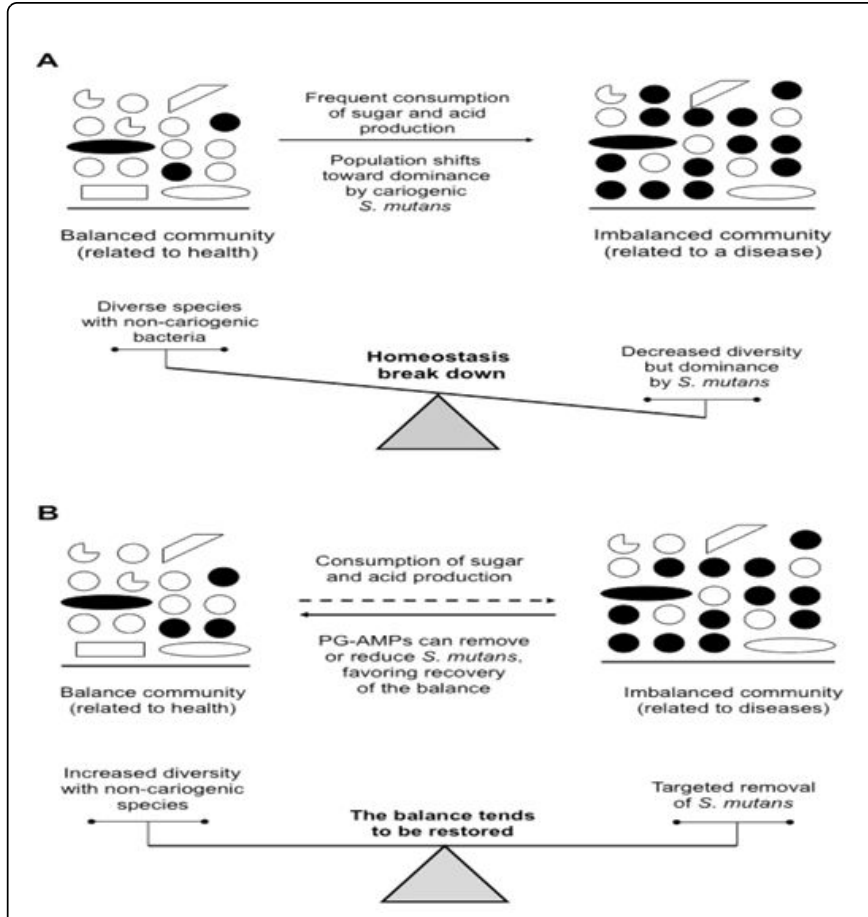

Figures 3 (A) and 3 (B): Schematic diagrams describe an example of an ecological factor to tip the balance of a dental biofilm community and another example of how PG-AMPs, such as HP30, IMB-2 and C16G2, regulate $S$. mutans to restore the balance of such a community. 3(A): Frequent consumption of dietary fermentable carbohydrates favors the overgrowth of sugar-fermentable and acidproducing-and-resistant bacteria (cariogenic pathogens), such as $S$. mutans (black circles) in dental biofilm. This likely results in a population shift toward dominance by $S$. mutans, which can reduce or eliminate many acid-sensitive, non-cariogenic bacteria (blank shapes) in the community, leading to the breakdown of the homeostasis and predisposing a site to tooth decay (dental caries). 3(B): Targeted removal of $S$. mutans (black circles) from the dental biofilm by using a PG-AMP, such as HP30, IMB-2 or C16G2, can eliminate or reduce $S$. mutans in the community. This targeted removal of the cariogenic pathogen may provide an advantage to non-cariogenic bacteria in the community, likely reversing the species diversity and restoring the homeostasis in the community that is less pathogenic. The diagrams are modified from Li et al. [3].

\section{Concluding Remarks}

Development of target-specific antimicrobial agents that selectively target specific pathogens may provide an opportunity to circumvent some clinical problems, such as ecological disruption and negative clinical consequences, due to the use of wide-spectrum antibiotics. Pheromone-guided antimicrobial peptides or PG-AMPs described in this review are proved to be effective to kill their target pathogens in in vitro model systems and hold the good potential for clinical application. Currently, efforts have been made to improve their efficacy and stability in physiologically relevant conditions of the human hosts, reducing their cytotoxicity, and incorporating them in novel formulates for clinical delivery. Because of their effectiveness, selectivity, relative stability and minimal toxicity towards the host cells, 
this new class of target-specific antimicrobial peptides has shown great potential for future development as alternatives to conventional antibiotics in fighting against certain types of infectious diseases.

\section{Acknowledgement}

This work was supported by CIHR Operating Grant MOP-115007 and by NSERC Discovery Grant RGPIN 311682-07. M.L. was the recipient of a Chinese Visiting Scholarship Award.

\section{References}

1. Marsh PD, Zaura E (2017) Dental biofilms: Ecological interactions in health and disease. J Clin Periodontol 44: S12-S22.

2. Kuramitsu HK, He X, Lux R, Anderson MH, Shi W (2007) Interspecies interactions within oral microbial communities. Microbiol Mol Rev 71: 653-670.

3. Li YH, Huang X, Tian XL (2017) Recent advances in dental biofilms: Impacts of microbial interactions on the biofilm ecology and pathogenesis. AIMS Bioengineering 4: 335-350.

4. Rosier BT, Marsh PD, Mira A (2018) Resilience of the oral microbiota in health: Mechanisms that prevent dysbiosis. J Dent Res 97: 371-380.

5. Ajdic D, McShan WM, McLaughlin RE, Savic G, et al. (2002) Genome sequence of Streptococcus mutans UA159, a cariogenic dental pathogen. Proc Natl Acad Sci 99: 14434-14439.

6. Mitchell TJ (2003) The pathogenesis of streptococcal infections: From tooth decay to meningitis. Nat Rev Microbiol 1:219-230.

7. Hogan D, Kolter R (2002) Why are bacteria refractory to antimicrobials? Curr Opin Microbiol 5: 472-7.

8. Hughes D, Anderson DI (2012) Selection of resistance at lethal and nonlethal antibiotic concentrations. Curr Opin Microbiol 15: 555-560.

9. Brogden NK, Brogden KA (2011) Will new generations of modified antimicrobial peptides improve their potential as pharmaceuticals? Int J Antimicrob Agents 38: 217-225.

10. Baltzer SA, Brown MH (2011) Antimicrobial peptides: promising alternatives to conventional antibiotics. J Mol Microbiol Biotechnol 20: 228-235.

11. Qiu XQ, Wang H, Lu XF, Zhang J, Li SF, et al. (2003) An engineered multi-domain bactericidal peptide as a model for targeted antibiotics against specific bacteria. Nat Biotechnol 21: 1480-1485.

12. Qiu XQ, Zhang J, Wang, Wu HGY (2005) A novel engineered peptide, a narrow-spectrum antibiotic, is effective against vancomycin-resistant Enterococcus faecalis. Antimicrob Agents Chemother 49: 1184-1189.

13. Eckert R. Qi F, Yarbrough DK, He J, Anderson MH et al. (2006) Adding selectivity to antimicrobial peptides: rational design of a multidomain peptide against Pseudomonas spp. Antimicrob Agents Chemother 50: $1480-1488$.

14. Franzman MR, Burnell KK, Dehkordi-Vakil FH, Guthmiller JM, Dawson DV et al. (2009) Targeted antimicrobial activity of a specific IgG-SMAP28 conjugate against Porphyromonas gingivalis in a mixed culture. Int $J$ Antimicrob Agents 33: 14-20.

15. Eckert R, He J, Yarbrough DK, Qi F, Anderson MH et al. (2006) Targeted killing of Streptococcus mutans by a pheromone-guided "smart" antimicrobial peptide. Antimicrob Agents Chemother 50: 3651-3657.
16. Mai J, Tian XL, Gallant JW, Merkley N, Biswas Z et al. (2011) A novel target-specific, salt-resistant antimicrobial peptide against the cariogenic pathogen Streptococcus mutans. Antimicrob Agents Chemother 55: 5205-5213.

17. He J, Eckert R, Pharm T, Simanian MD, Hu C, et al. (2007) Novel synthetic antimicrobial peptides against Streptococcus mutans. Antimicrob Agents Chemother 51: 1351-1358.

18. He J, Yarbrough DK, Kreth J, Anderson MH, Shi W, et al. (2010) Systematic approach to optimizing specifically targeted antimicrobial peptides against Streptococcus mutans. Antimicrob Agents Chemother 54: 2143-2151.

19. Patrzykat A, Gallant JW, Seo JK, Pytyck J, Douglas SE (2003) Novel antimicrobial peptides derived from flatfish genes. Antimicrob Agents Chemother 47: 2464-2470.

20. Syvitski RT, Tian XL, Sampara K, Salman A, Lee SF, et al. (2007) Structure-activity analysis of quorum-sensing signaling peptides from Streptococcus mutans. J Bacteriol 189: 1441-1450.

21. Tian XL, Chen C, Cyr K, Dong G, Salim H et al. (2017) Targeted killing of Streptococcus mutans in biofilms by a pheromone guided antimicrobial peptide HP30. J Antimicrob Res 1: 201.

22. Kaplan CW, Sim JH, Shah KR, Kolesnikova-Kaplan A, Shi WJ, et al. (2011) Selective membrane disruption: mode of action of C16G2, a specifically targeted antimicrobial peptide. Antimicrob Agents Chemother 55: 3446-3452.

23. Hossain MS, Biswas I (2012) An extracellular protease, SepM, generates functional competence-stimulating peptide in Streptococcus mutans UA159. J Bacteriol 194: 5886-5896.

24. Kalia VC (2013) Quorum sensing inhibitors: an overview. Biotechnol Adv 31: $224-245$.

25. LaSarre B, Federie MJ (2013) Exploiting quorum sensing to confuse bacterial pathogens. Microbiol Mol Biol Rev 77: 73-111.

26. Jenssen H, Hamill P, Hancock REW (2006) Peptide antimicrobial agents. Clin Microbiol Rev 19: 491-511.

27. Syeebny LM, Banoczy J, Baum BJ, Edgar WM, Epstein JB, et al. (1992) Saliva: its role in health and disease. Working Group 10 of the Commission on Oral Health, Research and Epidemiology (CORE) Int Dent J 42: 287-304.

28. Li L, He J, Eckert R, Yarbrough D, Lux R, et al. (2010) Design and characterization of an acid-activated antimicrobial peptide. Chem Biol Drug Des 75: 127-132.

29. Guo L, McLean JS, Yang Y, Eckert R, Kaplan CW, et al. (2015) Precisionguided antimicrobial peptide as a targeted modulator of human microbial ecology. Proc Natl Acad Sci 112: 7569-7574.

30. Edlund A, Yang Y, Hall AP, Guo L, Lux R, et al. (2013) An in vitro biofilm model system maintaining a highly reproducible species and metabolic diversity approaching that of the human oral microbiome. Microbiome 1: 25.

31. Sullivan R, Santarpia P, Lavender S, Gittins E, Liu Z, et al. (2011) Clinical efficacy of a specifically targeted antimicrobial peptide mouth rinse: targeted elimination of Streptococcus mutans and prevention of demineralization. Caries Res 45: 415-428. 